\title{
A response to 'A comment on geographically weighted regression with parameter-specific distance metrics'
}

\author{
Binbin Lu $\mathbb{D}^{\mathrm{a}}$, Chris Brunsdon ${ }^{\mathrm{b}}$, Martin Charlton ${ }^{\mathrm{b}}$ and Paul Harris $\mathbb{C}^{\mathrm{c}}$ \\ aSchool of Remote Sensing and Information Engineering, Wuhan University, Wuhan, China; ${ }^{\text {bNational }}$ \\ Centre for Geocomputation, Maynooth University, Maynooth, Co. Kildare, Ireland; 'Sustainable Soils and \\ Grassland Systems, Rothamsted Research, Okehampton, UK
}

\begin{abstract}
In this article, we respond to 'A comment on geographically weighted regression with parameter-specific distance metrics' by Oshan et al. (2019), published in this journal, where several concerns on the parameter-specific distance metric geographically weighted regression (PSDM GWR) technique are raised. In doing so, we review the developmental timeline of the multiscale geographically weighed regression modelling framework with related and equivalent models, including flexible bandwidth GWR, conditional GWR and PSDM GWR. In our response, we have tried to answer all the concerns raised in terms of applicability, veracity, interpretability and computational efficiency of the PSDM GWR model.
\end{abstract}

\section{ARTICLE HISTORY}

Received 13 February 2019

Accepted 18 February 2019

\section{KEYWORDS}

Multiscale; GWmodel; local regression; spatial heterogeneity; GWR

\section{Introduction}

We thank the authors of 'A comment on geographically weighted regression with parameter-specific distance metrics' (Oshan et al. 2019), for an interesting discussion of our work (Lu et al. 2017, 2018). The ideas raised are thought provoking and interesting. Having seen the paper, and read through the arguments presented, we feel that we would like to continue the discussion, and therefore thank the editors of IJGIS for allowing us to do so through this response. We choose to set this out as follows:

(A) The developmental timeline of multiscale geographically weighed regression (MGWR) together with related and equivalent models.

(B) Specific responses to each section of the comment.

(C) Concluding remarks.

\section{Description and timeline of MGWR and related models}

In its basic form, geographically weighed regression (GWR) calibrates a spatially-varying coefficient (SVC) model with local regressions at target locations, using nearby weighted data falling under a kernel at the center of each location (Brunsdon et al. 1996). Here, 
a single kernel bandwidth is used for its calibration, which is limiting in that it implicitly assumes the same degree of spatial smoothness for each of the SVCs. Thus, if some response to predictor variable relationships tend to operate at a larger-scale whilst other relationships operate at a smaller-scale, basic GWR will ignore these differences and only find a 'best-on-average' scale of relationship non-stationarity (as it uses only a single bandwidth). As a first step, to address this limitation, mixed GWR (also known as semiparametric GWR) can be implemented in which some relationships are assumed stationary (globally-fixed) whilst others are assumed non-stationary (spatially-varying) (Brunsdon et al., 1999, Mei et al. 2004, 2016, Nakaya et al. 2005). However, a mixed GWR only in part addresses the limitation, as the subset of spatially-varying relationships is still assumed to operate at the same spatial scale. Instead, MGWR can be used in which each relationship is specified using its own bandwidth, and in doing so, allows each relationship to vary at its own spatial scale.

The developmental timeline of MGWR where only Euclidean distances (EDs) were used follows that of Yang et al. (2011, 2012); Yang (2014), where it was called flexible bandwidth GWR (FBGWR); Leong and Yue (2017) ${ }^{1}$, where it was called conditional GWR (CGWR); and Fotheringham et al. (2017) ${ }^{2}$, where it was called multiscale GWR (MGWR). All articles implemented the idea of 'a vector of bandwidths' for GWR, as first set out in Brunsdon et al. (1999).

Yang et al. (2011) presented MGWR as exploratory tool to aid model building when choosing between simpler (basic and mixed) GWR models, while Yang et al. (2012) assessed the accuracy of the estimated MGWR coefficients through a simulation experiment. Leong and Yue (2017) provided a detailed study of MGWR through simulated and empirical case studies, where it was again promoted as an exploratory tool. Leong and Yue (2017) compared their implementation of MGWR with basic GWR and with locallylinear GWR (Wang et al. 2008) and found MGWR to perform the best, but with caveats with respect to negatively correlated predictors, computational costs and the number of predictors specified. Fotheringham et al. (2017) also provided a detailed study of MGWR through simulated and empirical studies.

Lu et al. $(2015,2017)^{3}$ provided another form of MGWR, but where MGWR could be implemented with both Euclidean distances (EDs) and non-Euclidean distances (NonEDs), which were themselves allowed to be specific to each response to predictor relationship. This model was named GWR with parameter-specific distance metrics (PSDM GWR) and is the subject of the comment that we are responding to. This variant of MGWR, when fitted using EDs for all relationships, provides an MGWR model that is very similar in design to those approaches listed above - all adapt concepts found in generalized additive models (GAMs) (Hastie and Tibshirani 1986, 1990).

Subsequent studies include that of Wolf et al. (2018) ${ }^{4}$ where the MGWR model of Fotheringham et al. (2017) was compared with an implementation of the Bayesian SVC model of Finlay (2011); and Murakami et al. (2019) ${ }^{5}$ where the MGWR model of Lu et al. (2017) (i.e. PSDM GWR with only EDs, termed FBGWR) was compared with the random effects eigenvector spatial filtering model of Murakami et al. (2017). In both instances, MGWR comported well with alternative SVC models that could similarly cater for scaledependent relationships. Lu et al. (2018) ${ }^{6}$ proposed methods to save on computational costs with PSDM GWR (some of which are pertinent to MGWR), together with a Minkowski approach to help guide distance metric choice. 
All MGWR models up to this juncture could provide a measure of (in-sample) model prediction accuracy, for example through the residual sum of squares (RSS). However, they could not provide a measure of model complexity, as separate weight matrices were found for each relationship due to the implementation of the back-fitting algorithm employed. This implied that it was difficult to compare MGWR to alternative regressions (e.g. ordinary least squares (OLS), basic or mixed GWR) in terms of Akaike Information Criterion (AIC). It also implied that inference in MGWR, in particular estimating meaningful SVC standard errors was not then developed. The only differences in MGWR outputs at this juncture (i.e. RSS, bandwidth-specific AICs, bandwidths and the SVCs themselves), would arise due to variations in the specification of the back-fitting algorithm, such as choice of coefficient starting values, levels of convergence. These choices could also dictate computational efficiency.

However, welcome advances by Yu et al. (2019) ${ }^{7}$ propose an inferential framework to MGWR through the calculation of a hat matrix, which in turn allows the calculation of a single AIC value for the whole model, together with the derivation of MGWR SVC standard errors, so that local pseudo $t$-tests can be implemented. Local pseudo $t$-tests are applied considering multiple hypothesis tests. Computational costs do increase with this advance.

In summary, MGWR, FBGWR, CGWR and PSDM GWR, effectively all refer to the same modelling framework, and any variant (say, with non-EDs), issue (say, the use of standardized data, see below) or advance (say, through an improved inferential framework) may be applicable to all of them.

\section{Specific responses}

We now respond to each section in turn of the comment to PSDM GWR (Oshan et al. 2019). We also revert to the naming conventions given in the PSDM GWR papers, which doesn't always make for easy reading, so clarity is given where needed.

\subsection{Response to the 'Introduction'}

We agree with the positive arguments made for MGWR, for which PSDM GWR provides a version. PSDM GWR is indeed a complex variant of MGWR and in this response, we present an argument for its value. One part of this we found puzzling, as it implies that Lu et al. (2016) is an implementation of PSDM GWR, which it is not. In fact, it is a study of basic GWR with Minkowski distances. Lu et al. (2017) introduce PSDM GWR, where it is developed further in Lu et al. (2018) to reduce computational overheads (and was not published in this journal). In addition, Lu et al. (2016) is cited as using the London house price (LHP) data while it actually uses simulated data.

\subsection{Response to the 'Background' section}

We feel there is a need for clarification on the timelines of the research topics reviewed here. For clarity, Lu et al. (2014a) investigate basic GWR with ED and non-ED metrics specifically road network distance (ND) and travel time (TT) and apply these GWR variants to the LHP data. Lu et al. (2016) again investigate basic GWR, but now with Minkowski distances through a simulation study. The PSDM GWR study of Lu et al. (2017) 
considers only ED and $\Pi T$ metrics for each relationship and is again evaluated with the LHP data. The second PSDM GWR study of Lu et al. (2018) was specifically focused on reducing computational overheads, not increasing them. Lu et al. (2018) re-investigated the use of ED and TT metrics for each relationship of the PSDM GWR model with the LHP data (i.e. from Lu et al. 2017), and demonstrated significant computational savings through a revised search strategy. We would also like to clarify that although Minkowski distances could be used in PSDM GWR, this is not essential. Any kind of distance metric is permissible.

In a separate analysis, Lu et al. (2018) investigated the use of Minkowski distances through the LHP data and a simulation experiment, where the objective was to assess the potential of Minkowski distances to guide distance metric choice in PSDM GWR. Given that computational costs could be saved through this revised search strategy, it was clearly stated that computational costs would increase with Minkowski distances over that found with known distance metrics such as $\Pi$, but that ultimately, computational costs could be reduced through parallel computing. Lu et al. (2018) provide the following statement to this effect. 'The PSDM GWR calibrations are expected to be time consuming, particularly when using the Minkowski approach. However, as each individual calibration is independent of each other, it would be straightforward to speed up the procedure by means of parallel computing (Harris et al. 2010b), but this is not assessed here.'

\subsection{Response to the 'Issues' section: 'Veracity and importance of the results'}

\subsubsection{Response to first point of contention}

In response to the first paragraph and Fig. 1 of the comment (Fig. 12 in Lu et al. 2018).

This is a valid point of contention, and we note the importance of reflecting on this. It may also promote further discussion on a number of other studies - the same simulation experiment is used in Yang et al. (2012), Yang (2014), Fotheringham et al. (2017) where in the latter study, similar small changes in RMSE are given in support of MGWR. However, this objection should be taken in perspective, as several other outputs are used to evaluate the proposed approach, in addition to this one.

In response to the second paragraph ${ }^{8}$ and Table 1 of the comment.

In all instances, the chronological sequence of investigated models from Lu et al. (2014a, 2017 , 2018) with the LHP data resulted in small improvements in R-squared (see details given in Table 1 of this response). However, the values are either included for information but not discussed (Lu et al. 2018) or reported with a caveat to their interpretation (Lu et al. 2014a, 2017). For example, Lu et al. (2017), state: 'Note however, that it is unwise to compare the three GWR models by their R-squared only, since values are directly affected by the bandwidths, which are not comparable when different distance metrics are used (see Lu et al. 2014a for further discussions)'.

The RSS values were reported in (Lu et al. 2017, 2018), but not in Lu et al. (2014a). The full sequence of RSS values is now reported in Table 1. In Lu et al. (2014a, 2017), the AICc values were correctly reported for OLS, basic GWR (GWR(ED)), and its variants GWR(ND) and GWR(TT). We assess the worth, if any, of bandwidth-specific or component-wise AICc values reported for the PSDM-GWR, FB-ED-GWR, FB-TT-GWR and BP-PSDM-GWR models from Lu et al. (2017, (2018) in response to the next point of contention. 


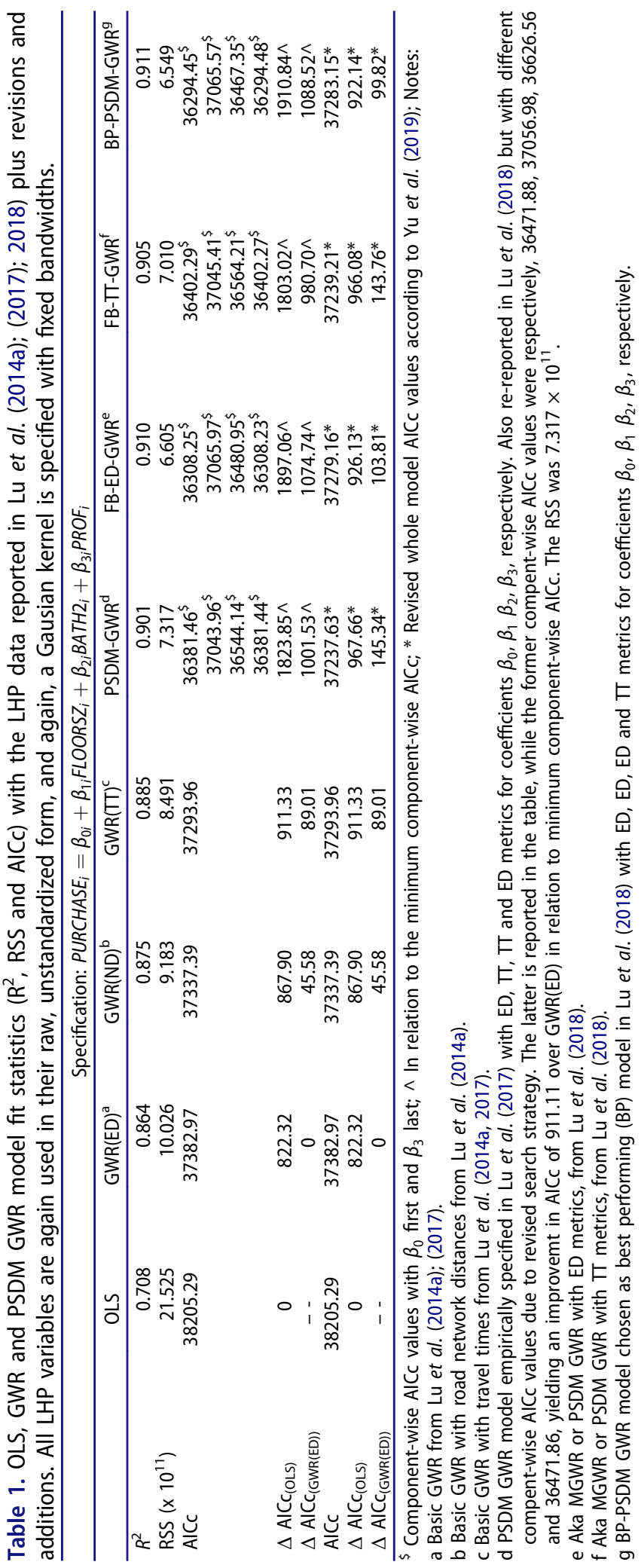




\subsubsection{Response to second point of contention}

Although, the work of Yu et al. (2019) is welcomed, it is stressed that an AICc for the whole model has been unavailable to all previous MGWR-type studies, not just those using PSDM GWR. We have added the option to calculate a whole model AICc value in the gwr.multiscale function of GWmodel (Lu et al. 2014b, Gollini et al. 2015) and the results are reported in Table 1.

From Table 1, it is clear that the use of (minimum) component-wise AICc values are not suitable to choose between different PSDM GWR constructions, or to choose a PSDM GWR model over an alternative, such as an OLS or basic GWR. Using this crude approach, the order of the poorest to best performing model was taken as follows: OLS, GWR(ED), GWR(ND), GWR(TT), FB-TT-GWR, PSDM-GWR, FB-ED-GWR and BP-PSDM-GWR. Thus the best performing model was initially taken as a PSDM GWR model with ED, ED, ED and TT metrics for coefficients $\beta_{0}, \beta_{1} \beta_{2}, \beta_{3}$, respectively. Using RSS to identify the best performing model, as recommended in Fotheringham et al. (2017) gives this sequence: OLS, GWR(ED), GWR(ND), GWR(TT), PSDM-GWR, FB-TT-GWR, FB-ED-GWR and BP-PSDM-GWR.

The now-available approach, with whole model AICc, gives this revised order of: OLS, GWR(ED), GWR(ND), GWR(TT), BP-PSDM-GWR, FB-ED-GWR, FB-TT-GWR and PSDM-GWR. The best performing model is now a PSDM GWR model with ED, $T$, $\Pi$ and ED metrics for coefficients $\beta_{0}, \beta_{1} \beta_{2}, \beta_{3}$, respectively. Furthermore, FB-TT-GWR (i.e. MGWR with TT metrics) out-performs FB-ED-GWR (i.e. a standard MGWR). Thus, the use of different distance metrics in this multiple bandwidth GWR framework appears to have greater value than that originally reported ${ }^{9,10}$.

\subsubsection{Response to third point of contention}

In response to Fig. 2 of the comment (Fig. 4 in Lu et al. 2017).

Fig. 2 of the comment compares coefficients from GWR(ED) (i.e. basic GWR), GWR(TT) (i.e. basic GWR with a TT metric) and PSDM-GWR with ED, TT, TT and ED metrics for coefficients $\beta_{0}, \beta_{1} \beta_{2}, \beta_{3}$, respectively. It does not contain a comparson of coefficients from FB-ED-GWR and FB-TT-GWR (i.e. corresponding MGWR models), which seems to be suggested by this point of contention. In this respect, we feel that the figure and its commentary in the article cited still hold. Note, Lu et al. (2018) do provide a comparison of FB-ED-GWR with FB-TT-GWR but with a different PSDMGWR specification, and thus provides a means to judge the influence of different MGWR-type model specifications.

\subsubsection{Response to fourth point of contention}

In response to this comment, no theoretical argument could be found for using standardized variables (mean $=0$ and standard deviation $=1$ ) in MGWR (or GAMs), or a practical demonstration of their value to MGWR, as opposed to unstandardized variables. The sentence 'Indeed, in other local spatial models, variance and bandwidth are known to be inseparable (Warnes and Ripley 1987, Zhang 2004)', references geostatistical studies not involving a bandwidth as a tuning parameter in moving window regression. We find the linkage made here puzzling since in geostatistical models variance is specified as a function of distance, and in any case, we are unsure as to why this implies data should be standardized. 
However, given this, we conducted an experiment on the effects of variable standardization in GWR and MGWR, where only EDs needed to be considered. We considered scaling (i.e. dividing variables by their standard deviation), centering (i.e. subtracting the mean of each variable) and standardizing (i.e. subtracting the mean and then dividing by the standard deviation). Generally, we found that the approaches involving centering (i.e. centering itself, and also standardizing) did lead to more reliable results (for example, when calibrating models via back-fitting where all bandwidths are equal in comparison to 'basic' GWR), but that scaling had little effect - for example, the scaled variables gave identical results to untransformed variables, and the centered variables gave identical results to the standardized variables. This will be reported in more detail, but a useful summary here might be that centering is useful, but scaling has little effect.

\subsubsection{Response to fifth point of contention}

In response to this comment, we first recall that Minkowski distances should not be viewed as a core component of PSDM GWR and second this comment discusses a simulation experiment that does not apply PSDM GWR, as Lu et al. (2016) only apply basic GWR. That said, the simulation experiment has merit providing insights into Minkowski distances with GWR. The experiment demonstrates that the choice of distance metric, not only influences the fit of GWR but also GWR's ability to accurately estimate known SVCs, and where outputs are dependent on the characteristics of the spatial process generated. Testing GWR with a random spatial process is appropriate, as GWR has been observed in some cases to imply structure in spatial data when none exists (Páez et al. 2011, Harris 2018); and a revised evaluation through simulations with known values of $p$ and $\theta$ would be extremely challenging. In the 'Discussion and concluding remarks' section of Lu et al. (2016), the value of GWR with Minkowski distances is discussed cautiously. Lu et al. (2016) state 'The Minkowski approach is not viewed as a negation of the standard GWR technique with EDs, but more a useful option, especially in urban applications of GWR.'

\subsection{Response to the 'Issues' section: 'Prediction versus inference'}

Following the timeline of MGWR-type models given in Section 1 of this response, all studies prior to Yu et al. (2019) had a reporting focus on 'in-sample' prediction accuracy often coupled with an assessment of parameter accuracy through a simulation experiment. This is common to the development of many GWR-based models (e.g. Wheeler 2007, Huang et al. 2010). Thus, Yang et al. (2011, 2012); Yang (2014), Leong and Yue (2017); Fotheringham et al. (2017); Wolf et al. (2018); Murakami et al. (2019) could be similarly criticized in this respect.

If a full assessment of prediction accuracy was an objective for any of these MGWRtype studies, and those of Lu et al. $(2015,2017,2018)$, then each could have followed an 'out-of-sample' approach as outlined in the GWR versus kriging studies of Harris et al. 2010a; Harris and Juggins 2011), but this was not a stated aim for these studies. However, given our experiments above with respect to use of data standardization and bandwidth estimation, MGWR may not estimate the one thing that it needs to do accurately to be considered a model capable of reliably identifying relationships operating at different spatial scales. Given our experiments found similar levels of 'in-sample' 
prediction accuracy for widely dissimilar bandwidths, then ultimately MGWR and variants thereof, may only be of real use as a spatial predictor (and future studies should properly evaluate this). This should not be surprising given GWR's roots lie in the local smoothing paradigm (Cleveland 1979, Loader 2004).

For inference in MGWR, the framework given in Yu et al. (2019) is one approach and is implicitly transferable to PSDM GWR. Another approach would be to transfer the bootstrap methodology outlined in Harris et al. (2017) but where the added computational burden would need addressing. However, inference in any GWR model is ultimately compromised in that no single model exists, and here the Bayesian SVC models have an advantage (Gelfand et al. 2003, Finlay, 2011). Bayesian SVC models identify the structure of non-stationary relationships operating at different spatial scales through a multivariate autocorrelation function, that unlike the analogous kernel functions used in any GWR-based model, are not parameterized by the model's in-sample prediction accuracy (at least with AIC).

\subsection{Response to the 'Issues' section: 'Tradeoff between interpretability and computational complexity'}

In response, note that computation savings for PSDM GWR are transferrable to MGWR. For example, PSDM GWR as described in Lu et al. (2017) uses an ad-hoc search strategy, which was revised with an improved search strategy in Lu et al. (2018). Recent experiments have revised the search strategy further - we intended to publish these reproducibly.

Again, although the rest of this section focusses on discussing Minkowski distance metrics with basic GWR, or with PSDM GWR, we expect much use to be in situations where the appropriate metric for the spatial process is contextually implied, such as road network distance in urban settings or river network distance in environmental settings relating to water resources.

\subsection{Response to the 'Conclusions' section}

Given the many names, from different publications, for essentially the same model, one name should be decided upon. We agree that MGWR provides the most appropriate over-arching name for this class of GWR models, reflecting a core remit of modelling non-stationary relationships, each potentially operating at their own spatial scales.

\section{Concluding remarks}

In the comment's 'Background' section, Phibbs and Luft (1995), Jones et al. (2010) and Carling et al. (2012) are all cited in support of the authors' assertion that '... the practical impacts of using non-ED metrics are often small'. The experiments of Boscoe et al. (2012) would also appear to be in line with this view. However, we should also note that studies in the UK and Japan (Martin et al., 1998, Martin et al. 2002, Okabe and Kitamura 2010) might suggest that this is not always the case. This leads us to wonder whether the issue may be context and experiment specific, and whether this might then influence the analyst's choices. The choice of distance metric ought to be theirs.

Early in 'Statistics for Spatial Data' Noel Cressie $(1993$, p. 6) notes that he represents locations with coordinates in a Euclidean space and uses Euclidean distances. He 
observes that other distances '... such as road miles or a composite measure that combines, say, travel expense, convenience and times...' may be appropriate. In a later section of the book he uses great circle distances. Non-Euclidean distances in an urban setting have been but one focus in GWR (Lu et al. 2014a); such metrics also turn up in other contexts. Curriero (2006) reports on the use of norm-dependent distances, as well as stream distances in a river network in spatial interpolation exercises. Similarly, Geodetic distances (Cressie et al. 1990) and water distances (Cressie and Majure 1997, Ver Hoef et al. 2006) have been specified. In a recent paper, Ver Hoef (2018) discusses metrics for linear networks, also in the context of spatial interpolation.

A related issue is in the computation of distances between polygonal spatial objects. Currently, in GWmodel, the location chosen to represent each polygon is its geometric centroid. This can give rise to some anomalies; for example, in the Republic of Ireland the centroid of Naas Rural Electoral Division (Ireland) lies inside the boundary for Naas Urban Electoral Division, as the latter area is entirely contained within the former. It may be that the population weighted centroids are appropriate - this remains an area for further research.

In our earliest (FORTRAN IV) GWR codes, we assumed that users would be working from data located in a planar coordinate system. We also assumed that suitable coordinates would be found to represent the centroids of polygonal regions if these were the units of analysis. In the early 2000s, this code was modified to allow analysts to use geographical coordinates in decimal degrees, computing great circle distances assuming a spherical earth with a radius of $6371 \mathrm{~km}$. Our view is that the provision of alternatives for the analyst gives flexibility over a wide range of possibilities. In GWmodel the default distances are Euclidean. Geodetic distances, if required, are computed for the WGS84 ellipsoid. The availability of other metrics may deter less advanced users. The question as to which distance is appropriate is clearly experiment dependent and for many users, Euclidean or geodetic may well suffice. This, however, remains an area for research. The choices should be there.

For MGWR, the size of each bandwidth directly acts on the scale of the corresponding coefficient estimates. Allowing each relationship's distance metric to also vary through PSDM GWR, refines the representation of the structural features of these spatially varying coefficient estimates. What is currently missing are demonstrations of PSDM GWR outside of the usual urban, hedonic price model setting, where each predictor of the model has a different, but natural distance metric. For example, a spatial study of species decline could be informed through a mixture of environmental, social and economic factors, requiring a wide variety of distance metrics - including say, water distances, transportation network distances and Euclidean distances.

\section{Notes}

1. Published on 31 March 2017.

2. Available on-line August 2017.

3. Available on-line 28 November 2016.

4. Available on-line 10 November 2017.

5. Available on-line as an arXiv version 26 September 2017 and available on-line as a journal version 20 December 2018.

6. Available on-line 7 April 2018. 
7. Available on-line as a pre-print version 8 May 2018 and available on-line as a journal version 22 January 2019.

8. The comment again incorrectly references Lu et al. (2016) as using the LHP data. It only used simulated data.

9. Table 1 only reports on whole model AICc values for four of a possible 16 combinations investigated in Lu et al. (2018), meaning that any one of 12 alternative PSDM GWR models could provide a lower AICc, still.

10. The revised search strategy for PSDM GWR reported in Lu et al. (2018) still holds, as it was firmly based on the component-wise AICc values.

\section{Disclosure statement}

No potential conflict of interest was reported by the authors.

\section{Funding}

This work was supported by the National Natural Science Foundation of China [41871287, U1833201];UK Biotechnology and Biological Sciences Research Council Grant [BBSRC BB/ J004308/1];Open Research Fund Program of Shenzhen Key Laboratory of Spatial Smart Sensing and Services Shenzhen University [1712].

\section{Notes on contributors}

Binbin Lu is a Lecturer in the School of Remote Sensing and Information Engineering, Wuhan University, Wuhan, Hubei, China. His research interests include spatial statistics, geographically weighted modeling, spatial data analysis, open-source geographic information systems, and $\mathrm{R}$ coding.

Chris Brunsdon is Professor of Geocomputation and Director of the National Centre for Geocomputation, National University of Ireland Maynooth, Maynooth, Kildare, Ireland. His research interests include spatial data analysis and statistical modeling, computational data science, spatial data visualization, and the analysis of social and economic data.

Martin Charlton is Senior Lecturer in the National Centre for Geocomputation, Maynooth University, Maynooth, County Kildare, Ireland. His research interests include spatial modeling, quantitative geography, geographic information science, and spatial epidemiology.

Paul Harris is a Senior Research Scientist at Rothamsted Research, North Wyke, Okehampton, Devon EX20 2SB, UK. His research focuses on the development and application of spatial statistics to agricultural, ecological, and environmental data.

\section{ORCID}

Binbin Lu (1) http://orcid.org/0000-0001-7847-7560

Paul Harris (i) http://orcid.org/0000-0003-0259-4079

\section{References}

Boscoe, F.P., Henry, K.A., and Zdeb, M.S., 2012. A nationwide comparison of driving distance versus straight-line distance to hospitals. The Professional Geographer, 64 (2), 188-196. doi:10.1080/ 00330124.2011 .583586 
Brunsdon, C., Fotheringham, A.S., and Charlton, M., 1999. Some notes on parametric significance tests for geographically weighted regression. Journal of Regional Science, 39 (3), 497-524. doi:10.1111/0022-4146.00146

Brunsdon, C., Fotheringham, A.S., and Charlton, M.E., 1996. Geographically weighted regression: a method for exploring spatial nonstationarity. Geographical Analysis, 28 (4), 281-298. doi:10.1111/j.1538-4632.1996.tb00936.x

Carling, K., Han, M., and Håkansson, J., 2012. Does Euclidean distance work well when the p-median model is applied in rural areas? Annals of Operations Research, 201 (1), 83-97. doi:10.1007/s10479-012-1214-2

Cleveland, W.S., 1979. Robust locally weighted regression and smoothing scatterplots. Journal of the American Statistical Association, 74 (368), 829-836. doi:10.1080/01621459.1979.10481038

Cressie, N., 1993. Statistics for Spatial Data. New York: John Wiley \& Sons, Inc.

Cressie, N. and Majure, J., 1997. Non-point source pollution of surface waters over a watershed. In: V. Barnett and K. Turkman, eds. Statistics for the environment 3: pollution assessment and control. New York: New York, 201-224.

Cressie, N., Gotway, C.A., and Grondona, M.O., 1990. Spatial prediction from networks. Chemometrics and Intelligent Laboratory Systems, 7 (3), 251-271. doi:10.1016/0169-7439(90)80115-M

Curriero, F.C., 2006. On the use of Non-Euclidean Distance measures in geostatistics. Mathematical Geology, 38 (8), 907-926. doi:10.1007/s11004-006-9055-7

Finley, A.O., 2011. Comparing spatially-varying coefficients models for analysis of ecological data with non-stationary and anisotropic residual dependence. Methods in Ecology and Evolution, 2 (2), 143-154. doi:10.1111/j.2041-210X.2010.00060.x

Fotheringham, A.S., Yang, W., and Kang, W., 2017. Multiscale Geographically Weighted Regression (MGWR). Annals of the American Association of Geographers, 107 (6), 1247-1265. doi:10.1080/ 24694452.2017.1352480

Gelfand, A.E., et al., 2003. Spatial modeling with spatially varying coefficient processes. Journal of the American Statistical Association, 98 (462), 387-396. doi:10.1198/016214503000170

Gollini, I., et al., 2015. GWmodel: an R Package for exploring spatial heterogeneity using geographically weighted models. Journal of Statistical Software, 63 (17), 1-50. doi:10.18637/jss.v063.i17

Harris, P., et al., 2010a. The use of geographically weighted regression for spatial prediction: an evaluation of models using simulated data sets. Mathematical Geosciences, 42 (6), 657-680. doi:10.1007/s11004-010-9284-7

Harris, P., et al., 2017. Introducing bootstrap methods to investigate coefficient non-stationarity in spatial regression models. Spatial Statistics, 21, 241-261. doi:10.1016/j.spasta.2017.07.006

Harris, P., 2018. A simulation study on specifying a regression model for spatial data: choosing between autocorrelation and heterogeneity effects. Geographical Analysis, 30.

Harris, P. and Juggins, S., 2011. Estimating freshwater acidification critical load exceedance data for Great Britain using space-varying relationship models. Mathematical Geosciences, 43 (3), 265-292. doi:10.1007/s11004-011-9331-z

Harris, R., et al., 2010b. Grid-enabling geographically weighted regression: a case study of participation in higher education in England. Transactions in GIS, 14 (1), 43-61. doi:10.1111/ tgis.2010.14.issue-1

Hastie, T. and Tibshirani, R., 1986. Generalized additive models. Statistical Science, 1 (3), 297-310. doi:10.1214/ss/1177013604

Hastie, T. and Tibshirani, R., 1990. Generalized additive models. London: Chapman and Hall/CRC.

Huang, B., Wu, B., and Barry, M., 2010. Geographically and temporally weighted regression for modeling spatio-temporal variation in house prices. International Journal of Geographical Information Science, 24 (3), 383-401. doi:10.1080/13658810802672469

Jones, S.G., et al., 2010. Spatial implications associated with using Euclidean Distance Measurements and Geographic Centroid Imputation in Health Care Research. Health Services Research, 45 (1), 316-327. doi:10.1111/j.1475-6773.2009.01044.x

Leong, Y.-Y. and Yue, J.C., 2017. A modification to geographically weighted regression. International Journal of Health Geographics, 16 (1), 11. doi:10.1186/s12942-017-0085-9 
Loader, C., 2004. Smoothing: local regression techniques. Berlin: Center for Applied Statistics and Economics (CASE), Humboldt-Universit, 1-30.

Lu, B., et al., 2014a. Geographically weighted regression with a non-Euclidean distance metric: a case study using hedonic house price data. International Journal of Geographical Information Science, 28 (4), 660-681. doi:10.1080/13658816.2013.865739

Lu, B., et al., 2014b. The GWmodel R package: further topics for exploring spatial heterogeneity using geographically weighted models. Geo-Spatial Information Science, 17 (2), 85-101. doi:10.1080/10095020.2014.917453

Lu, B., et al., 2015. Calibrating a geographically weighted regression model with parameter-specific distance metrics. Procedia Environmental Sciences, 26, 109-114. doi:10.1016/j. proenv.2015.05.011

Lu, B., et al., 2016. The Minkowski approach for choosing the distance metric in geographically weighted regression. International Journal of Geographical Information Science, 30 (2), 351-368. doi:10.1080/13658816.2015.1087001

Lu, B., et al., 2017. Geographically weighted regression with parameter-specific distance metrics. International Journal of Geographical Information Science, 31 (5), 982-998. doi:10.1080/ 13658816.2016 .1263731

Lu, B., et al., 2018. Improvements to the calibration of a geographically weighted regression with parameter-specific distance metrics and bandwidths. Computers, Environment and Urban Systems, 71, 41-57. doi:10.1016/j.compenvurbsys.2018.03.012

Martin, D., et al., 1998. Geographical aspects of the uptake of renal replacement therapy in England. International Journal of Population Geography, 4 (3), 227-242.

Martin, D., et al., 2002. Increasing the sophistication of access measurement in a rural healthcare study. Health \& Place, 8 (1), 3-13. doi:10.1016/S1353-8292(01)00031-4

Mei, C.-L., He, S.-Y., and Fang, K.-T., 2004. A note on the mixed geographically weighted regression model. Journal of Regional Science, 44 (1), 143-157. doi:10.1111/j.1085-9489.2004.00331.x

Mei, C.-L., Xu, M., and Wang, N., 2016. A bootstrap test for constant coefficients in geographically weighted regression models. International Journal of Geographical Information Science, 30 (8), 1622-1643. doi:10.1080/13658816.2016.1149181

Murakami, D., et al., 2017. A Moran coefficient-based mixed effects approach to investigate spatially varying relationships. Spatial Statistics, 19, 68-89. doi:10.1016/j.spasta.2016.12.001

Murakami, D., et al., 2019. The importance of scale in spatially varying coefficient modeling. Annals of the American Association of Geographers, 109 (1), 1-21.

Nakaya, T., et al., 2005. Geographically weighted Poisson regression for disease association mapping. Statistics in Medicine, 24 (17), 2695-2717. doi:10.1002/sim.2129

Okabe, A. and Kitamura, M., 2010. A computational method for market area analysis on a network. Geographical Analysis, 28 (4), 330-349.

Oshan, T., et al., 2019. A comment on geographically weighted regression with parameter-specific distance metrics. International Journal of Geographical Information Science, 1-12. doi:10.1080/ 13658816.2019.1572895

Páez, A., Farber, S., and Wheeler, D., 2011. A simulation-based study of geographically weighted regression as a method for investigating spatially varying relationships. Environment and Planning A, 43 (12), 2992-3010. doi:10.1068/a44111

Phibbs, C.S. and Luft, H.S., 1995. Correlation of travel time on roads versus straight line distance. Medical Care Research and Review, 52 (4), 532-542. doi:10.1177/107755879505200406

Ver Hoef, J.M., 2018. Kriging models for linear networks and non-Euclidean distances: cautions and solutions. Methods in Ecology and Evolution, 9 (6), 1600-1613. doi:10.1111/2041-210X.12979

Ver Hoef, J.M., Peterson, E., and Theobald, D., 2006. Spatial statistical models that use flow and stream distance. Environmental and Ecological Statistics, 13 (4), 449-464. doi:10.1007/s10651006-0022-8

Wang, N., Mei, C.L., and Yan, X.D., 2008. Local linear estimation of spatially varying coefficient models: an improvement on the geographically weighted regression technique. Environment and Planning A, 40 (4), 986-1005. doi:10.1068/a3941 
Warnes, J.J. and Ripley, B.D., 1987. Problems with likelihood estimation of covariance functions of spatial Gaussian processes. Biometrika, 74 (3), 640-642. doi:10.1093/biomet/74.3.640

Wheeler, D.C., 2007. Diagnostic tools and a remedial method for collinearity in geographically weighted regression. Environment and Planning A, 39 (10), 2464-2481. doi:10.1068/a38325

Wolf, L.J., Oshan, T.M., and Fotheringham, A.S., 2018. Single and multiscale models of process spatial heterogeneity. Geographical Analysis, 50 (3), 223-246. doi:10.1111/gean.v50.3

Yang, W., 2014. An extension of geographically weighted regression with flexible bandwidths. (Doctoral). St Andrews.

Yang, W., Fotheringham, A.S., and Harris, P., 2011. Model selection in GWR: the development of a flexible bandwidth GWR. The 11th International Conference on GeoComputation. London, UK, 1-7.

Yang, W., Fotheringham, A.S., and Harris, P., 2012. An extension of geographically weighted regression with flexible bandwidths. GISRUK 2012. Lancaster, 1-7.

$\mathrm{Yu}, \mathrm{H}$., et al., 2019. Inference in multiscale geographically weighted regression. Geographical Analysis. doi:10.1111/gean.12189

Zhang, H., 2004. Inconsistent estimation and asymptotically equal interpolations in model-based geostatistics. Journal of the American Statistical Association, 99 (465), 250-261. doi:10.1198/ 016214504000000241 
Copyright of International Journal of Geographical Information Science is the property of Taylor \& Francis Ltd and its content may not be copied or emailed to multiple sites or posted to a listserv without the copyright holder's express written permission. However, users may print, download, or email articles for individual use. 